\title{
Inverse Modeling of Asian Dust Emissions with POPC Observations: A TEMM Dust Sand Storm 2014 Case Study
}

\author{
Keiya Yumimoto ${ }^{1, *}$, Itsushi Uno ${ }^{2}$, Xiaole Pan $^{2, * *}$, Tomoaki Nishizawa ${ }^{3}$, \\ Sang-Woo Kim ${ }^{4}$, and Nobuo Sugimoto ${ }^{3}$ \\ ${ }^{1}$ Meteorological Research Institute, Japan Meteorological Agency, Ibaraki, Japan \\ ${ }^{2}$ Research Institute for Applied Mechanics, Kyushu University, Fukuoka, Japan \\ ${ }^{3}$ National Institute for Environmental Studies, Ibaraki, Japan \\ ${ }^{4}$ School of Earth and Environmental Sciences, Seoul National University, Seoul, Korea
}

\begin{abstract}
An inverse modeling system for estimating Asian dust emissions was developed by combining the GEOS-Chem chemical transport model with the Green's function method. We applied the system to two heavy dust storms that occurred in 2014 (10-25 March and 24 May to 5 June), using surface-based polarization optical particle counter (POPC) observations at Fukuoka. Validation by independent observation datasets, including POPC measurements and $\mathrm{PM}_{10}$ observations at Seoul, showed that the use of a posteriori dust emissions improved overestimations in the $a$ priori simulation and achieved much better agreement with observations. Satellite observations, surface synoptic observations, and modeled wind fields indicated that the major dust source region differed between the two dust storms; the major dust outbreak of one storm occurred in the northeastern Gobi Desert, whereas that of the other occurred in the southern Gobi Desert. The a posteriori dust emissions successfully reproduced this difference. Thus, the inverse modeling system developed in this study was able to improve the estimation of not only the intensity but also the geographical distribution of dust emissions.
\end{abstract}

(Citation: Yumimoto, K., I. Uno, X. L. Pan, T. Nishizawa, S.-W. Kim, and N. Sugimoto, 2017: Inverse modeling of Asian dust emissions with POPC observations: A TEMM dust sand storm 2014 case study. SOLA, 13, 31-35, doi:10.2151/sola.2017006.)

\section{Introduction}

In East Asia, one of the major aerosols is soil dust, which has large impacts on air quality, human health, and radiative balance. Dust particles are generally swept up from the surface by strong winds over deserts, and the emission amount strongly depends on land-surface parameters such as soil texture and wetness, vegetation, and snow cover. In numerical chemical transport models (CTMs), independent dust emission schemes (e.g., Marticorena and Bergametti 1995; Shao et al. 2004) are widely used to represent the emission process. However, the complicated dependence of the dust emission process on land-surface conditions makes accurate estimation of the distribution and intensity of dust emissions difficult. In fact, a dust model intercomparison project (Uno et al. 2006) found that dust emission amounts estimated by eight CTMs showed large variation, sometimes differing by a factor of ten. This result indicates that there is considerable uncertainty in the estimation of dust emissions.

To reduce the uncertainty, considerable effort has gone into

Corresponding author: Keiya Yumimoto, Research Institute for Applied Mechanics, Kyushu University, Kasuga, Fukuoka 816-8580, Japan. E-mail: yumimoto@riam.kyushu-u.ac.jp.

"Present affiliation: Research Institute for Applied Mechanics, Kyushu University, Fukuoka, Japan

"** Present affiliation: Institute of Atmospheric Physics, Chinese Academy of Sciences, Beijing, China

(C)2017, the Meteorological Society of Japan. the development of inverse modeling systems. For example, Maki et al. (2011) used Bayesian synthesis inversion and surface $\mathrm{PM}_{10}$ concentration data to optimize dust emission fluxes in the Gobi desert, and Yumimoto and Takemura (2015) performed an 8-year inverse modeling study of Asian dust with a global aerosol transport model that used a four-dimensional variational assimilation method and satellite-measured aerosol optical thickness (AOT).

In this study, we developed an inverse modeling system for dust emissions based on GEOS-Chem CTM (Bey et al. 2001) and the Green's function method and applied it to two heavy dust storms that occurred in 2014, one during 10-25 March (Period A) and the other during the period from 20 May to 5 June (Period B). These two storms were selected as a case study of dust and sand storm (DSS) events by working group-1 (WG1) of the Tripartite Environment Ministers Meeting among China, Japan, and Korea (TEMM) (http://www.temm.org). Dust surface concentrations measured by a polarization optical particle counter (POPC) were used as the observational constraint. To our best knowledge, POPC is the only instrument that can measure mass concentration of dust aerosols in near real-time. We investigated the characteristics of the two dust storms by using a posteriori dust emissions that had been independently validated with POPC measurements and surface $\mathrm{PM}_{10}$ concentrations shared by TEMM.

\section{Data and study method}

We used GEOS-Chem version 09-02 to simulate the dust storms. GEOS-Chem is driven by assimilated meteorological data provided by GEOS-5. Global simulations by GEOS-Chem have a horizontal resolution of $2^{\circ} \times 2.5^{\circ}$ and 47 vertical layers. To represent the finer distribution of dust aerosols, we performed a high-resolution, one-way-nested grid simulation covering South and East Asia with a horizontal resolution of $0.5^{\circ} \times 0.667^{\circ}$ and 47 vertical layers.

GEOS-Chem uses four bins for mineral dust with radii of $0.1-$ 1.0 (DST1), 1.0-1.8 (DST2), 1.8-3.0 (DST3), and 3.0-6.0 (DST4) $\mu \mathrm{m}$, and it employs a dust emission scheme (Fairlie et al. 2007) that combines an entrainment scheme (Zender et al. 2003) with a source function (Ginoux et al. 2004). In the dust emission scheme, the total vertical dust flux $(F)$ is represented by

$$
F=\beta\left(1-A_{\mathrm{s}}\right) S \alpha Q_{\mathrm{s}},
$$

where $A_{\mathrm{s}}$ is the fractional area of snow cover, $\alpha$ is the sandblasting mass efficiency, $Q_{\mathrm{s}}$ is the horizontal saltation flux parameterized by the friction velocity and threshold friction velocity (White 1979), and $S$ is the source function, which is the product of the fractional area of land subject to dust emission and erodibility. We introduced a scaling factor $\beta$ to adjust the total vertical dust flux $(F)$ in the inverse modeling procedure.

The basic settings of the dust emission scheme, including the source function, were designed for global simulations conducted for the TRACE-P and ACE-Asia aircraft campaigns (Fairlie et al. 2007). Therefore, we applied some modifications for application to more recent dust storms or for finer nested simulations. The land-use map was updated by using United States Geological 
a) a posteriori dust emission (Period $A)$

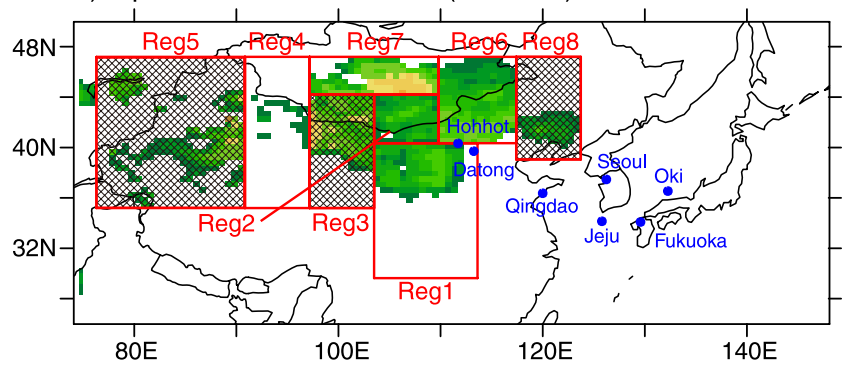

b) a posteriori dust emission (Period B)

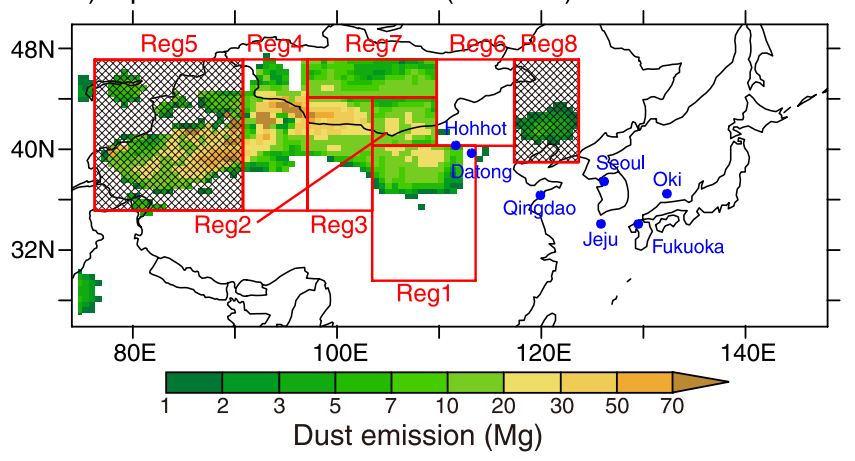

Fig. 1. Horizontal distributions of a posteriori dust emissions during periods A (10-25 March) and B (20 May to 5 June). Red boxes define the eight subregions. Hatched boxes indicate those subregions in which the reduction rate of analysis error was smaller than $10 \%$. Blue circles show locations of observation sites.
Survey Land Cover Type data. We used the dust particle size distribution proposed by Zhang et al. (2013) for the size-resolved mass fractions of dust emissions instead of the distribution used in the standard GEOS-Chem model. Following Feng (2007), we optimized wet scavenging efficiencies in each size bin to prevent unrealistically large dust depositions.

We employed the Green's function method (Yumimoto et al. $2014,2016)$. We defined a quadratic cost function to represent the distance between modeled and observed concentrations and then adjusted $\beta$ to minimize the cost function. The major dust source region of East Asia was divided into eight subregions (Reg1Reg8; Fig. 1), and the scaling factors $(\beta)$ for each subregion were inversely optimized. To find the minimum of the cost function by adjusting $\beta$, source-receptor (S-R) relationships are required. We performed eight sensitivity simulations in which dust emissions from each subregion were decreased by $20 \%$. Figures $2 \mathrm{~d}$ and $2 \mathrm{~h}$ show the contributions of dust emissions from each subregion to the total concentration as calculated by using the S-R relationships. Consequently, we ran 10 simulations altogether: an a priori simulation, the eight sensitivity simulations, and an a posteriori simulation with the optimized dust emissions. As the background error, we assigned a value of $50 \%$ of the a priori emission.

Two POPCs were in operation at Fukuoka and Seoul during the dust events. A POPC uses a linearly polarized laser beam at a wavelength of $780 \mathrm{~nm}$ to measure the number concentration of aerosol particles sampled via an inlet and their diameters over a range of $0.1-1.0 \mu \mathrm{m}$. The measured number concentration is distributed into four size bins corresponding to the GEOS-Chem dust bins. We converted the measured number concentrations of the DST2-DST4 bins to mass concentrations by using the typical density for Asian dust $\left(2520 \mathrm{~kg} \mathrm{~m}^{-3}\right)$, under the assumption that dust particles were dominant in the coarser bins, and used the 2-hour a) POPC (DST2) at Fukuoka (Period A)

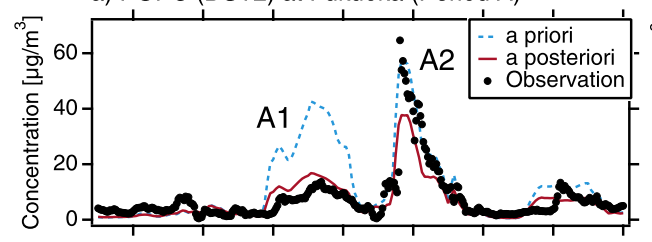

b) POPC (DST2) at Seoul (Period A)

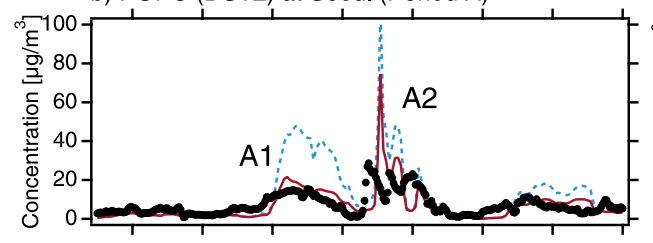

c) PM10 at Seoul (Period A)

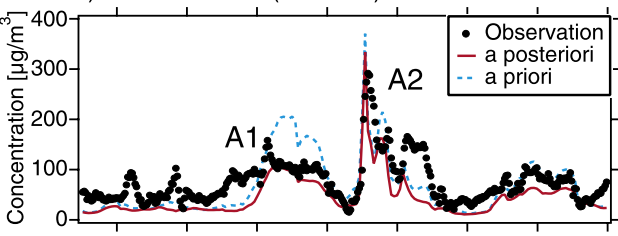

d) Contribution (DST2) at Fukuoka (Period A)

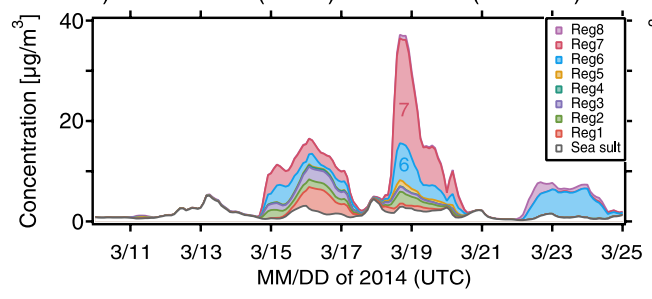

e) POPC (DST2) at Fukuoka (Period B)

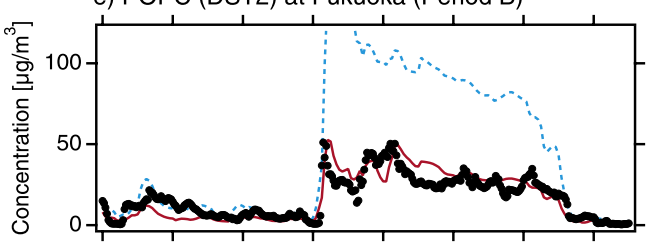

f) POPC (DST2) at Seoul (Period B)

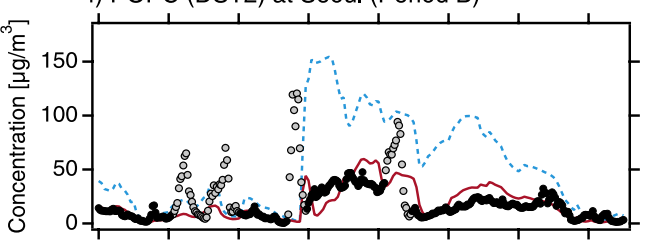

g) PM10 at Seoul (Period B)

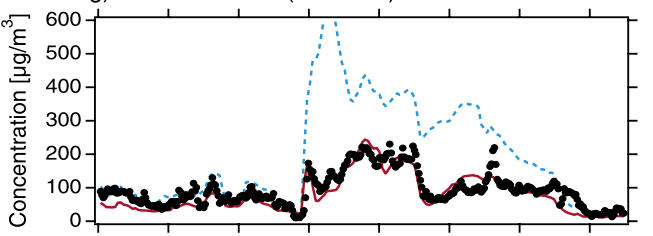

h) Contribution (DST2) at Fukuoka (Period B)

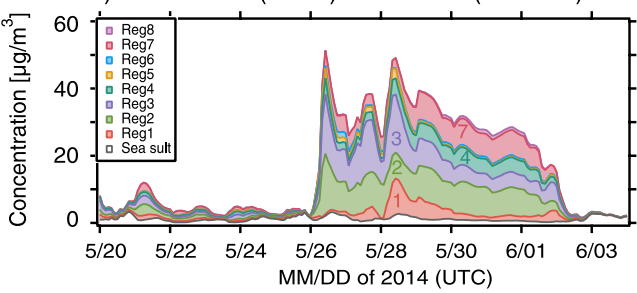

Fig. 2. Time series of (a, e) DST2 concentrations at Fukuoka, (b, f) DST2 concentrations at Seoul, (c, g) $\mathrm{PM}_{10}$ concentrations at Seoul, and (d, h) contributions of the eight subregions to the DST2 concentrations at Fukuoka. Left panels, Period A; right panels, Period B. Gray circles in (f) represent data that were eliminated from the analysis because of possible misidentification of fine rain and fog drops as aerosol particles. Observation site locations are shown in Fig. 1. 
averaged mass concentrations of DST2 and DST3 at Fukuoka for the inverse modeling. POPC observations at Seoul were used for independent validation of the inverse modeling results. As the minimum absolute error, we used $15 \mu \mathrm{g} \mathrm{m}^{-3}$, which was derived from the standard deviations of the observed concentrations during the targeted periods. We then defined the observation error as $15+0.3 C_{\mathrm{o}} \mu \mathrm{g} \mathrm{m}^{-3}$, where $C_{\mathrm{o}}$ is the observed mass concentration, by taking into account the backward scattering signal uncertainty (about 15\%) and uncertainties due to the number-tomass concentration conversion, to mixing of other species, and to representational and transport errors. The details of the POPC measurements have been described elsewhere (Kobayashi et al. 2014; Pan et al. 2015). Measurements during 10-25 March and from 20 May to 5 June were used as observational constraints for Period A and B, respectively.

Ground-based $\mathrm{PM}_{10}$ observations are shared in TEMM. Hourly $\mathrm{PM}_{10}$ concentrations measured at Korean, Chinese, and Japanese sites were used for validation of the inverse modeling results. To detect dust storms over the source region, we used aerosol index (AI) from the Ozone Monitoring Instrument onboard the Aura (Torres et al. 2002) and SYNOP (surface synoptic observations) present weather codes ( $\mathrm{ww}=06,07,08,09,30-35$, and 98). The distribution of the monthly Normalized Difference Vegetation Index (NDVI) from the MODIS (Moderate Resolution Imaging Spectroradiometer) in the MOD13C2.006 product (Didan 2015) was also used. Definition of statistical measures used in validation is provided in the Supplement 1.

\section{Results and discussion}

During Period A, the passage of two dust storms was detected, on 15-16 March (A1) and 18-19 March (A2), at Fukuoka and Seoul (Figs. 2a, 2b, and 2c). In general, the modeled concentrations obtained without inverse modeling (hereafter the a priori concentrations) overestimated the observed concentrations.

During Period B, higher DST2 and $\mathrm{PM}_{10}$ concentrations (Figs. $2 \mathrm{e}, 2 \mathrm{~g}$, and $2 \mathrm{~g}$ ) that lasted about one week were observed. The $500 \mathrm{hPa}$ geopotential height distribution and cloud images (Fig. $\mathrm{S} 1)$ clearly show that the slow eastward motion of a high-pressure system along the meandering jet stream was responsible for the persistently high dust concentrations. The a priori concentrations during Period $\mathrm{B}$ were almost double the observed concentrations.

During Period A, the concentrations simulated with the optimized dust emissions (hereafter, the a posteriori concentrations) were improved compared with the overestimated a priori concentrations (Figs. 2a, 2b, and 2c); they showed good agreement with the observed concentrations, particularly during A1. During A2, the $a$ posteriori concentrations underestimated the observed DST2 concentrations at Fukuoka, whereas they showed better agreement with the observed DST2 and $\mathrm{PM}_{10}$ concentrations at Seoul. At both sites, most statistical scores of the a posteriori concentrations with respect to the observed DST2 concentrations were better than those of the a priori concentrations (Supplementary Table S1). Inverse modeling also improved most statistical scores with respect to the observed $\mathrm{PM}_{10}$ concentrations at Seoul, although the negative normalized mean bias of the a posteriori concentrations was larger than that of the a priori concentrations. Underestimated a posteriori concentrations on 19 and 22-23 March were responsible for this degradation.

During Period B, the inverse modeling considerably improved the overestimated a priori concentrations (Figs. 2e, $2 \mathrm{f}$, and $2 \mathrm{~g}$ ). The a posteriori concentrations successfully reproduced the peak concentration at the beginning of the dust event, the persistently high concentrations during the event, and the end time of the dust event at Fukuoka (Fig. 2e). The overestimation of DST2 and $\mathrm{PM}_{10}$ concentrations at Seoul was also improved by the inverse modeling (Figs. 2f, 2g; Table S2). Both the a priori and a posteriori concentrations failed to capture two abrupt peaks on 25 and 28 May in the observed DST2 concentrations. However, neither peak appeared in the $\mathrm{PM}_{10}$ observations. Similar unrealistic peaks occasionally seen in POPC observations at Seoul can be explained by the misidentification of fine rain and fog drops taken into the instrument as aerosol particles. After comparing the DST2 concentrations with cloud and rain detected by lidar observations at Seoul (Fig. S2), we excluded observations of possible rain and fog droplets (gray circles in Fig. 2f) from the analysis. Better statistical scores for Seoul observations (Table S2) confirmed that the $a$ posteriori emissions improved the dust concentrations estimated in the downwind regions. In particular, the correlation coefficient $(0.87)$ and the index of agreement $(0.92)$ of the a posteriori concentrations were high with respect to the $\mathrm{PM}_{10}$ concentrations.

Comparisons with other Chinese, Korean, and Japanese $\mathrm{PM}_{10}$ observations (Fig. S3) showed that, compared with a priori emissions, a posteriori dust emissions achieved better agreement with observed concentrations at both Chinese, Korean and Japanese sites; thus, inverse modeling improved dust emissions during both periods $\mathrm{A}$ and $\mathrm{B}$. The propagation of the arrival time of dust storms in the observations was also successfully reproduced in the simulations.

Figure 1 shows the horizontal distribution of the a posteriori dust emissions, and the optimized $\beta$ and dust emission amounts for each subregion are listed in Table 1 . The analysis error $(\mathbf{P})$ and the reduction rate of analysis error $(R R A E)$ were calculated as follows:

$$
\begin{aligned}
& \mathbf{P}=\left(\mathbf{Q}^{-1}+\mathbf{G}^{T} \mathbf{R}^{-1} \mathbf{G}\right)^{-1}, \\
& R R A E=\operatorname{trace}(\mathbf{Q}-\mathbf{R}) / \operatorname{trace}(\mathbf{Q}) \times 100,
\end{aligned}
$$

where $\mathbf{Q}$ and $\mathbf{R}$ are the background and observation error covariance matrices. $\mathbf{G}$ is the Green's function matrix and calculated by the S-R simulations. $T$ denotes transpose. The difference between the analysis and background errors in Eq. (2) indicates the magnitude of the impact of the observations on an a posteriori solution. Thus, a smaller RRAE corresponds to a smaller observational constraint. We excluded those subregions in which the $R R A E$ was smaller than $10 \%$ from the analyses.

To resolve the overestimation in the a priori concentrations, the inverse modeling reduced dust emissions in almost all subregions during both periods. Dust emissions were particularly reduced in Reg1, Reg2, and Reg4 during Period A and in Reg2, Reg3, Reg6, and Reg7 during Period B. Total dust emission amounts were about halved during both periods (Table 1).

To investigate changes in the horizontal distribution of dust emission, we calculated the relative dust emission contribution from each of the eight subregions to the total dust emission amount (Fig. 3). In Period A, although the inverse modeling reduced the contribution of Reg4 significantly, the contributions of Reg6 and Reg7 increased, and together they accounted for 70\% of the total dust emissions (Fig. 3a). Therefore, the inverse modeling shifted the major dust source from the western to the northeastern Gobi Desert. During A2, the contributions of Reg6 and Reg7 were much larger than those of the other subregions, accounting for $67.5-77.7 \%$ (Fig. 2d). Different from Period A, during Period

Table 1. Optimization of dust emissions in the eight subregions. Values for subregions in which the reduction rate of analysis error was less than $10 \%$ are shown in italics.

\begin{tabular}{lccccccc}
\hline & \multicolumn{3}{c}{ Period A } & & \multicolumn{2}{c}{ Period B } \\
\cline { 2 - 3 } \cline { 6 - 7 } & $\beta$ & \multicolumn{2}{c}{ Emissions (Tg) } & & \multicolumn{2}{c}{ Emissions (Tg) } \\
\cline { 3 - 4 } \cline { 6 - 7 } & & a priori & a posteriori & & a priori & a posteriori \\
\hline Total & & 12.44 & 6.14 & & 31.41 & 17.23 \\
Reg1 & 0.41 & 1.29 & 0.54 & & 0.54 & 2.60 & 1.43 \\
Reg2 & 0.21 & 1.45 & 0.30 & & 0.38 & 3.01 & 1.14 \\
Reg3 & 0.62 & 2.09 & 1.29 & & 0.45 & 5.99 & 2.69 \\
Reg4 & 0.03 & 2.78 & 0.08 & & 0.66 & 6.42 & 4.23 \\
Reg5 & 1.02 & 1.00 & 1.02 & & 0.99 & 6.30 & 6.23 \\
Reg6 & 0.52 & 2.05 & 1.07 & & 0.02 & 4.41 & 0.09 \\
Reg7 & 1.03 & 1.65 & 1.70 & & 0.49 & 2.55 & 1.25 \\
Reg8 & 0.90 & 0.15 & 0.14 & & 1.04 & 0.15 & 0.16 \\
\hline
\end{tabular}

$\beta$, scaling factor 

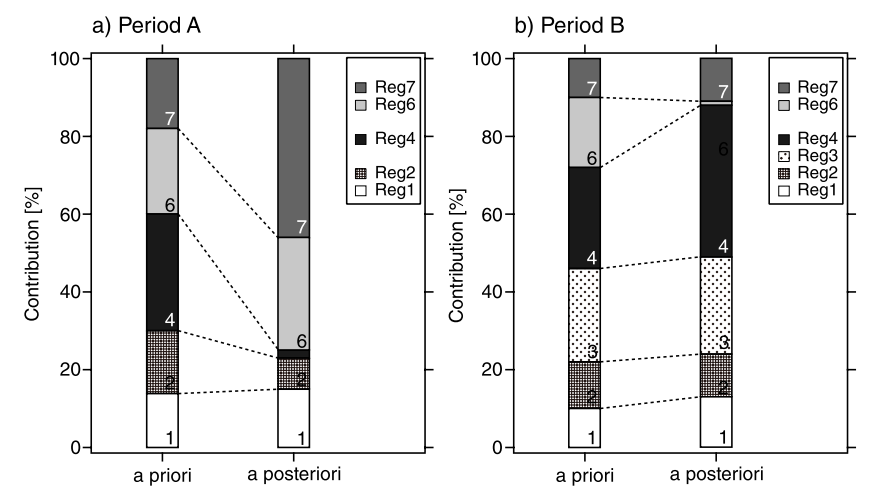

Fig. 3. Comparison of the dust emission contributions of the subregions (excluding those in which the reduction rate of analysis error was less than $10 \%$ ) relative to total emissions during (a) Period A and (b) Period B.

$\mathrm{B}$ the inverse modeling reduced the contribution of Reg6 and increased the contributions of Reg1, Reg3, and Reg4 (Fig. 3b); these changes shifted the major dust source to the southern Gobi Desert. Reg2-Reg4 accounted for $57.0-88.7 \%$ during Period B (Fig. 2h).

We performed a comprehensive analysis of the observed and modeled datasets to investigate conditions in the source region. On 16 March, during Period A (Fig. 4a), a center of low pressure was at $46^{\circ} \mathrm{N}, 113^{\circ} \mathrm{E}$, and strong westerly and northwesterly winds were produced over Reg2, Reg6, and Reg7. In Reg2, Reg6, and Reg7, AI was $>1$, and SYNOP detected dust storms in Reg2, Reg6, and Reg7. Thus, the northeastern Gobi Desert was the major source region. The distribution of the a posteriori AOT showed good agreement with dust emissions indicated by the AI and SYNOP detection results. In contrast, on 24 May 2014 during Period B (Fig. 4b), strong surface winds $\left(>10 \mathrm{~m} \mathrm{~s}^{-1}\right)$ associated with a low pressure at $46^{\circ} \mathrm{N}, 115^{\circ} \mathrm{E}$ blew across Reg1-3 and Reg6. AI values greater than 1 were widely distributed, and SYNOP

a) Period A (16 March 2014)

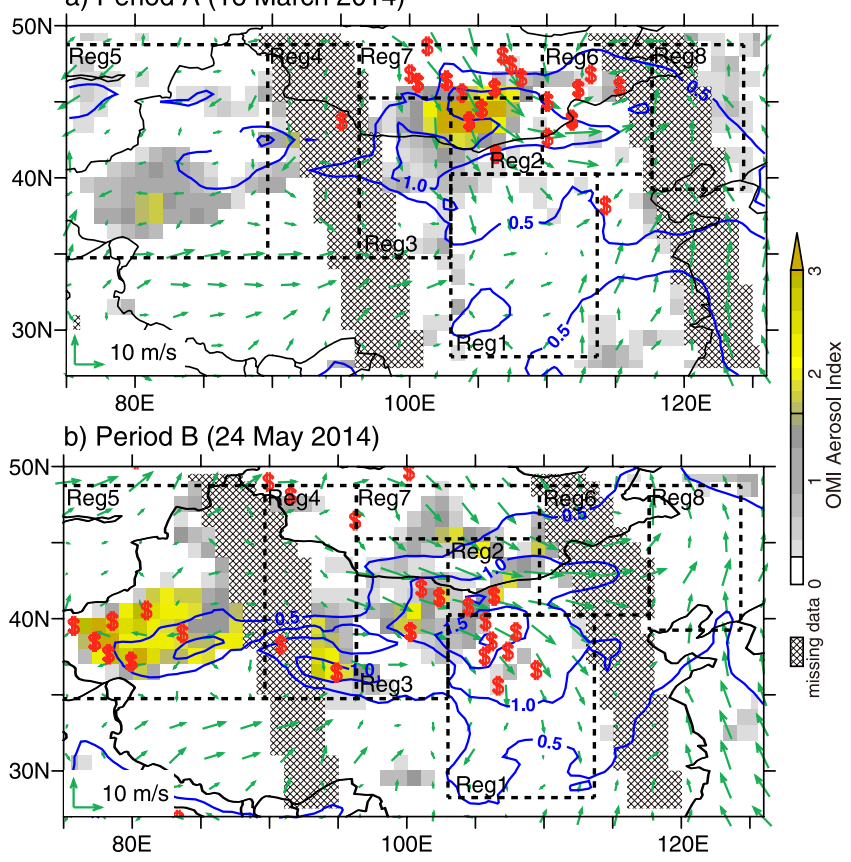

Fig. 4. Horizontal distributions of Aerosol Index values of the Ozone Monitoring Instrument (scale on the right), modeled wind (green vectors), modeled aerosol optical thickness (blue contours), and SYNOP dust observations (red \$ symbols) on (a) 16 March (Period A) and (b) 24 May (Period B) 2014 . detected a dust storm in Reg1-4. Thus, the southern Gobi Desert was the major source region. The a posteriori AOT distribution was consistent with the observations.

The comprehensive analysis revealed that the major source region was different between Period A and Period B. SYNOP dust detection suggested that dust storms occurred in Reg2 and Reg6-7 (northeastern Gobi Desert) during Period A, and in Reg1-4 (southern Gobi Desert) during Period B. The distributions of the a posteriori $\mathrm{AOT}$ and emissions were consistent with the SYNOP observations and successfully reproduced the source regions difference. Kim et al. (2013) suggested that potential of using NDVI for dust mobilization in the GOCART model. We used satellite-measured NDVI values to investigate changes in the vegetation cover. The regionally averaged NDVI in Reg6 increased substantially from 0.16 to 0.22 between Period A (March) and Period B (May), and in Reg7 it also increased substantially from 0.14 to 0.22 between Period A and Period B. The increases in the NDVI in Reg2 (from 0.089 to 0.095 ), and Reg3 (from 0.10 and 0.13 ) were slight, and the NDVI stayed low. The increased and relatively large NDVI values in Reg6 and Reg7 can explain the smaller dust emission contributions of these subregions in Period B relative to their contributions in Period A, and the southwestward shift of the dust source. These findings show that the inverse modeling system can not only improve the estimation of the amount of dust emissions but also determine their distribution appropriately.

\section{Concluding remarks}

We developed an inverse modeling system for the optimization of Asian dust emissions based on GEOS-Chem and the Green's function method. The system divided the main dust source region in East Asia into eight subregions and optimized dust emissions in each region by using observational constraints. We then performed inverse modeling experiments for two heavy dust storms in 2014 using surface dust concentration data observed by POPC at Fukuoka.

The inverse modeling results were validated with several observation datasets, including $\mathrm{PM}_{10}$ concentrations shared by the TEMM WG1, and POPC measurements at Seoul. The a posteriori dust emissions reduced overestimates in the a priori simulations and achieved much better agreement with the surface observations. Statistical analyses showed that most error statistics of the a posteriori concentrations were better than those of the a priori concentrations.

AI values, SYNOP dust detection, and the modeled wind fields revealed that the major dust source region differed between the two dust storms; dust particles were mainly emitted from the northeastern Gobi Desert during Period A and from the southern Gobi Desert during Period B. The a posteriori dust emissions successfully reproduced this source difference, indicating that the inverse modeling system is capable of improving estimations of not only the amount but also the distribution of dust emissions.

\section{Acknowledgments}

This work was supported by the Japan Society for the Promotion of Science (JSPS) KAKENHI Grant Numbers JP25220101, JP26701004, JP15K05296, and JP16H02946, the Environment Research and Technology Development Fund (No. S-12) of the Japanese Ministry of the Environment, and the KMA R\&D program under Grant KMIPA 2015-2011. This research paper is a contribution to the Joint Research on Dust and Sandstorms project of Working Group 1 of the Tripartite Environment Ministers Meeting.

Edited by: O. Wild 


\section{Supplements}

Supplement 1 presents the statistics used for the validation. Supplemental Tables S1 and S2 show the error statistics for the observed versus modeled concentrations during Period A and Period B, respectively. Figure S1 shows the geopotential height at $500 \mathrm{hPa}$. Figure S2 shows a time series of the DST2 concentration and a time-height plot of the extinction coefficient at Seoul. Figure S3 shows time series of PM10 concentrations at Chinese, Korean, and Japanese sites.

\section{References}

Bey, I., J. Jacob, R. M. Yantosca, A. Logan, B. D. Field, A. M. Fiore, Q. Li, H. Y. Liu, J. Mickley, and M. G. Schultz, 2001: Global modeling of tropospheric chemistry with assimilated meteorology: Model description and evaluation. J. Geophys. Res., 106, 73-95.

Didan, K., 2015: MOD13C2 MODIS/Terra Vegetation Indices Monthly L3 Global 0.05Deg CMD V006. NASA EOSDIS Land Processes DAAC, doi:10.5067/MODIS/MOD13C2. 006.

Fairlie, T., D. J. Jacob, and R. J. Park, 2007: The impact of transpacific transport of mineral dust in the United States. Atmos. Environ., 41, 1251-1266, doi:10.1016/j.atmosenv.2006.09. 048.

Feng, J., 2007: A 3-mode parameterization of below-cloud scavenging of aerosols for use in atmospheric dispersion models. Atmos. Environ., 41, 6808-6822, doi:10.1016/j.atmosenv. 2007.04.046.

Ginoux, P., J. M. Prospero, O. Torres, and M. Chin, 2004: Long-term simulation of global dust distribution with the GOCART model: correlation with North Atlantic Oscillation. Environ. Model. Softw., 19, 113-128, doi:10.1016/ S1364-8152(03)00114-2.

Kalnay, E., M. Kanamitsu, R. Kistler, W. Collins, D. Deaven, L. Gandin, M. Iredell, S. Saha, G. White, J. Woollen, Y. Zhu, A. Leetmaa, R. Reynolds, M. Chelliah, W. Ebisuzaki, W. Higgins, J. Janowiak, K. C. Mo, C. Ropelewski, J. Wang, R. Jenne, and D. Joseph, 1996: The NCEP/NCAR 40-Year Reanalysis Project. Bull. Amer. Meteor. Soc., 77, 437-471.

Kim, D., M. Chin, H. Bian, Q. Tan, M. E. Brown, T. Zheng, R. You, T. Diehl, P. Ginoux, and T. Kucsera, 2013: The effect of the dynamic surface bareness on dust source function, emission, and distribution, J. Geophys. Res. Atmos., 118, 871-886, doi:10.1029/2012JD017907.

Kobayashi, H., M. Hayashi, K. Shiraishi, Y. Nakura, T. Enomoto, K. Miura, H. Takahashi, Y. Igarashi, H. Naoe, N. Kaneyasu, T. Nishizawa, and N. Sugimoto, 2014: Development of a polarization optical particle counter capable of aerosol type classification. Atmos. Environ., doi:10.1016/j.atmosenv. 2014.05.006.

Maki, T., T. Y. Tanaka, T. T. Sekiyama, and M. Mikami, 2011: The impact of ground-based observations on the inverse technique of aeolian dust aerosol. SOLA, 7A, 21-24, doi: 10.2151/sola.7A-006.

Marticorena, B., and G. Bergametti, 1995: Modeling the atmospheric dust cycle: 1. Design of a soil-derived dust emission scheme. J. Geophys. Res., 100, 16415, doi:10.1029/95JD 00690.

Pan, X., I. Uno, Y. Hara, M. Kuribayashi, H. Kobayashi, N. Sugimoto, S. Yamamoto, T. Shimohara, and Z. Wang, 2015: Observation of the simultaneous transport of Asian mineral dust aerosols with anthropogenic pollutants using a POPC during a long-lasting dust event in late spring 2014. Geophys. Res. Lett., 42, 1593-1598, doi:10.1002/2014GL 062491.

Shao, Y., 2004: Simplification of a dust emission scheme and comparison with data. J. Geophys. Res., 109, 1-6, doi: 10.1029/2003JD004372.

Torres, O., P. K. Bhartia, J. R. Herman, A. Sinyuk, P. Ginoux, and B. Holben, 2002: A long-term record of aerosol optical depth from TOMS observations and comparison to AERONET measurements. J. Atmos. Sci., 59, 398-413, doi: 10.1175/1520-0469(2002)059<0398:ALTROA>2.0.CO;2.

Uno, I., Z. Wang, M. Chiba, Y. S. Chun, S. L. Gong, Y. Hara, E. Jung, S.-S. Lee, M. Liu, M. Mikami, S. Music, S. Nickovic, S. Satake, Y. Shao, Z. Song, N. Sugimoto, T. Tanaka, and D. L. Westphal, 2006: Dust model intercomparison (DMIP) study over Asia: Overview. J. Geophys. Res., 111, doi: 10.1029/2005JD006575.

White, B. R., 1979: Soil transport by winds on Mars. J. Geophys. Res., 84, 4643, doi:10.1029/JB084iB09p04643.

Yumimoto, K., and T. Takemura, 2015: Long-term inverse modeling of Asian dust: Interannual variations of its emission, transport, deposition, and radiative forcing. J. Geophys. Res. Atmos., 120, 1582-1607, doi:10.1002/2014JD022390.

Yumimoto, K., I. Uno, and S. Itahashi, 2014: Long-term inverse modeling of Chinese $\mathrm{CO}$ emission from satellite observations. Environ. Pollut., 195, 308-318, doi:10.1016/j.envpol. 2014.07.026.

Yumimoto, K., Y. Morino, T. Ohara, Y. Oura, M. Ebihara, H. Tsuruta, and T. Nakajima, 2016: Inverse modeling of the 137Cs source term of the Fukushima Dai-ichi Nuclear Power Plant accident constrained by a deposition map monitored by aircraft. J. Environ. Radioact., 164, 1-12, doi: 10.1016/j.jenvrad.2016.06.018.

Zender, C. S., H. Bian, and D. Newman, 2003: Mineral Dust Entrainment and Deposition (DEAD) model: Description and 1990s dust climatology. J. Geophys. Res., 108, 4416, doi:10.1029/2002JD002775.

Zhang, L., J. F. Kok, D. K. Henze, Q. Li, and C. Zhao, 2013: Improving simulations of fine dust surface concentrations over the western United States by optimizing the particle size distribution. Geophys. Res. Lett., 40, 3270-3275, doi: 10.1002/grl.50591.

Manuscript received 19 December 2016, accepted 10 February 2017 SOLA: https://www.jstage.jst.go.jp/browse/solal 